\title{
GROUP OBJECT STRUCTURE AND STATE ESTIMATION IN THE PRESENCE OF MEASUREMENT ORIGIN UNCERTAINTY
}

\author{
Lyudmila Mihaylova and Amadou Gning \\ Lancaster University, Dept. of Communication Systems, InfoLab21, Lancaster LA1 4WA, UK \\ Email: mila.mihaylova@lancaster.ac.uk,e.gning@lancaster.ac.uk
}

\begin{abstract}
This paper proposes a technique for motion and group structure estimation of moving targets based on evolving graph networks [1] in the presence of measurement origin uncertainty. The proposed method, through an evolving graph model, allows to jointly estimate the group target and the group structure with the uncertainty. The performance of the algorithm is evaluated and results with real ground moving target indicator data are presented.
\end{abstract}

Index Terms - Evolving graphs, random graphs, group target tracking, nonlinear estimation, Monte Carlo methods, data association.

\section{INTRODUCTION}

Group object tracking has been investigated during the last years in various applications including road traffic systems, military surveillance and in particular for ground moving target indicator (GMTI) tracking [2] and robotics applications $[3,4,5,6]$. GMTI group target tracking is concerned with tracking groups of objects, such as convoys of vehicles, moving on the ground from an airborne sensor platform, taking noisy measurements, e.g., range and bearing angle.

Groups of targets can be considered as formations of entities whose number varies over time because targets can enter a scene, or disappear at random times. The groups can split, merge, to be near to each other or move largely independently on each other. However, it is typical for group formations to maintain some patterns of movement [7] and hence the methods for group tracking differ from the methods of standard multiple-target tracking. Although individual targets in the group can exhibit independent movement at a certain level, overall the group will move as one whole, synchronising the movement of the individual entities and avoiding collisions.

Different models of groups of objects have been proposed in the literature, such as the flocking models $[8,9,10]$, in-

We acknowledge the support of the EPSRC, under project EP/E027253/1 and the support of the UK MOD Data and Information Fusion Defence Technology Centre. We are thankful also QinetiQ, Malvern for providing us with the real ground moving target indicator data sets and useful discussions. Finally, this work is completed with stimulating discussions and networking activities with the EU COST action TU0702. cluding leader-follower models [7]. However, estimating the dynamic evolution of the group structure has not been widely studied in the literature, although there are similarities with methods used in evolving network models [11, 12].

In [1], the group structure is modeled as evolving undirected random graphs with measurements which origin is exactly known. Then, an evolution model is defined for the group structure and the efficiency of the approach is showed through a scenario with simulated data. The main contribution of the present paper is the proposed solution for group object structure and state estimation with measurement uncertainty. A Joint Probabilistic Data Association Filter (JPDAF) is applied by taking into account the structure of the group. Results are presented over real GMTI data.

The remaining part of the paper is organised in the following way. Section 2 formulates group structure modeling with evolving random graphs and the group structure evolution model. Section 3 presents the Bayesian formulation an implementation solution based on a particle filter and joint probability data association. Section 4 presents the results and finally Section 5 summarises the results.

\section{GROUP STRUCTURE MODELING}

\subsection{Evolving Network Models}

One of the challenges in group object tracking is in the necessity of updating the group structure and modeling the interactions between separate components. In [1], at time instant $t$, a group structure $\boldsymbol{G}_{t}$ is defined as a graph. The group structure $\boldsymbol{G}_{t}$ models both the targets within the groups (nodes in the graph) and some relations between the group members, which is reflected by the edges between the related graph nodes. Each node of the graph corresponds to an object within the group and the presence (resp. absence) of an edge between two nodes means presence (absence) of interactions between these objects.

\subsection{Group Object Structure Evolution Model}

In [1], an evolution model $\boldsymbol{G}_{t}=f\left(\boldsymbol{G}_{t-1}, \boldsymbol{X}_{t}\right)$ for the group structure, for time $t>0$ and an initialisation process $\boldsymbol{G}_{0}=$ $f\left(\boldsymbol{X}_{0}\right)$ for time $t=0$ are introduced. The vector $\boldsymbol{X}_{t}=$ 
$\left(\boldsymbol{x}_{t, 1}, \ldots, \boldsymbol{x}_{t, n}\right)$ comprises the state vectors of all the targets and $f$ denote the desired evolution model.

The system

$\left\{\begin{array}{l}t=0, \boldsymbol{G}_{0}=f_{I}\left(\boldsymbol{X}_{0}\right), \\ t>0, \boldsymbol{G}_{t}=f_{N S} \circ f_{N I} \circ f_{E U}\left(\boldsymbol{G}_{t-1}, \boldsymbol{X}_{t}\right),\end{array}\right.$

shows the decomposition of the evolution model $f$ according to the time $t$ and according to three distinctive steps: edge updating, node adding and node removal where $\circ$ denotes the composition operation; $f_{I}$ is an Initialisation model; $f_{E U}$ is the graph Edge Updating model; $f_{N I}$ is the graph Nodes Incorporation; $f_{N S}$ is the graph Nodes Suppression model.

The edge updating step and the nodes incorporation step are modeled using a second graph $G^{\prime}$ having the group centres as nodes. Indeed, the computational complexity can be reduced when some information about group centres (means, variances and the distances between them) is used. We refer to [1], for more details.

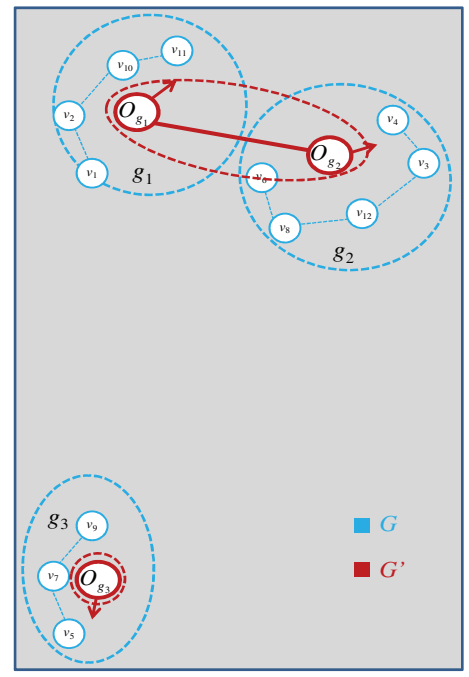

Fig. 1. Illustration of the graphical approach for the group structure. In this example the graph structure $G$ contains three connected components (three groups). A second graph $G^{\prime}$ models the distance between groups. $G^{\prime}$ is used for the edge updating process, the node incorporation process and in the data association process

\section{BAYESIAN FORMULATION AND PARTICLE FILTER IMPLEMENTATION}

Each target $i$ is characterised by its state vector $\boldsymbol{x}_{t, i}=\left(x_{t, i}\right.$, $\left.\dot{x}_{t, i}, y_{t, i}, \dot{y}_{t, i}\right)^{\prime}$ (comprising the positions $x_{t, i}, y_{t, i}$ and velocities $\dot{x}_{t, i}, \dot{y}_{t, i}$ in $x$ and $y$ directions respectively); ' denotes the transpose operation. At each time instant, the set of objects tracked in a group $\boldsymbol{g}$ can be modeled by a Random Finite Set (RFS, see [7]) $\boldsymbol{X}_{t}^{\boldsymbol{g}}$.

At time $t$ a measurement vector $z_{t}$ is received which can be described as a function of the augmented RFS state $\boldsymbol{X}_{t}=$
$\left\{\boldsymbol{X}_{t}^{\boldsymbol{g}_{1}}, \ldots, \boldsymbol{X}_{t}^{\boldsymbol{g}_{n_{G}}}\right\}$ ( $n_{G}$ denotes the number of groups in $\boldsymbol{G}_{\boldsymbol{t}}$ and $\boldsymbol{g}_{i}, i \in\left\{1, \ldots, n_{G}\right\}$ denotes the groups in $\boldsymbol{G}_{\boldsymbol{t}}$. Assuming that the measurement likelihood function $p\left(\boldsymbol{z}_{t} \mid \boldsymbol{X}_{t}\right)$ can be calculated, the purpose is to compute sequentially the state probability density function for each group of objects. Additionally, the groups' movements are assumed independent. Indeed, the changes of the groups such as merging and splitting are taken into account during the graph update process.

Under the Markovian assumption for the state transition, the Bayesian prediction and filtering steps can be written as follows:

$$
\begin{gathered}
p\left(\boldsymbol{X}_{t}, \boldsymbol{G}_{t} \mid \boldsymbol{Z}_{1: t-1}\right)=p\left(\boldsymbol{G}_{t} \mid \boldsymbol{X}_{t}, \boldsymbol{Z}_{1: t-1}\right) \times p\left(\boldsymbol{X}_{t} \mid \boldsymbol{Z}_{1: t-1}\right) \\
=\int p\left(\boldsymbol{G}_{t} \mid \boldsymbol{X}_{t}, \boldsymbol{G}_{t-1}\right) \times \\
p\left(\boldsymbol{X}_{t} \mid \boldsymbol{X}_{t-1}, \boldsymbol{G}_{t-1}\right) p\left(\boldsymbol{X}_{t-1}, \boldsymbol{G}_{t-1} \mid \boldsymbol{Z}_{1: t-1}\right) d \boldsymbol{X}_{t-1} d \boldsymbol{G}_{t-1} \\
p\left(\boldsymbol{X}_{t}, \boldsymbol{G}_{t} \mid \boldsymbol{Z}_{1: t}\right)=\frac{p\left(\boldsymbol{z}_{t} \mid \boldsymbol{X}_{t}, \boldsymbol{G}_{t}\right) \times p\left(\boldsymbol{X}_{t}, \boldsymbol{G}_{t} \mid \boldsymbol{Z}_{1: t-1}\right)}{p\left(\boldsymbol{z}_{t} \mid \boldsymbol{Z}_{1: t-1}\right)}
\end{gathered}
$$

where $Z_{1: t}$ is the set of measurements up to time $t$ and $z_{t}$ is the current vector of measurements. With the independence assumptions between groups, the following supplementary equation

$$
p\left(\boldsymbol{X}_{t} \mid \boldsymbol{X}_{t-1}, \boldsymbol{G}_{t-1}\right)=\prod_{\boldsymbol{g}_{i} \in \boldsymbol{G}_{t-1}} p\left(\boldsymbol{X}_{t}^{\boldsymbol{g}_{i}} \mid \boldsymbol{X}_{t-1}^{\boldsymbol{g}_{i}}\right),
$$

can be written.

In order to perform the correction step, the likelihood function $p\left(\boldsymbol{z}_{t} \mid \boldsymbol{X}_{t}, \boldsymbol{G}_{t}\right)$ of the whole state has to be evaluated by means of a data association approach. The JPDA algorithm [13] is used to resolve the measurement origin uncertainty.

In addition other information about the graph structure can be used, such as the distance between groups in order to reduce the number of hypotheses. Figure 1 shows an example where group $\boldsymbol{g}_{3}$ can be considered separately from groups $\boldsymbol{g}_{1}$ and $\boldsymbol{g}_{2}$. The graph $\boldsymbol{G}_{t}^{\prime}$ estimated at each time and used in the edge updating and in the nodes incorporation steps can be also used to reduce the data association computation. At each time instant, groups in the same graph $G_{t}^{\prime}$ 's connected component are gathered in the same data association process: the graph $\boldsymbol{G}_{t}^{\prime}$ offers a straightforward method of clustering the targets for the data association process.

Denote by $\left\{\boldsymbol{g}_{1}^{\prime}, \ldots, \boldsymbol{g}_{n_{G^{\prime}}}^{\prime}\right\}$, the set of $n_{G^{\prime}}$ connected components in graph $G^{\prime}$. In other words, any connected component $\boldsymbol{g}_{i}^{\prime}$ models a set of groups that are close enough to be treated in a same data association algorithm. Under independence assumption between the $\boldsymbol{g}_{i}^{\prime}$, the following equation (5) can be written.

$$
\begin{gathered}
p\left(\boldsymbol{z}_{t} \mid \boldsymbol{X}_{t}, \boldsymbol{G}_{t}\right)=p\left(\boldsymbol{z}_{t} \mid \boldsymbol{X}_{t}, \boldsymbol{G}_{t}, \boldsymbol{G}_{t}^{\prime}\right) \\
=\prod_{i=1, \ldots, n_{G^{\prime}}} p\left(\boldsymbol{z}_{t}^{\boldsymbol{g}_{i}^{\prime}} \mid \boldsymbol{X}_{t-1}^{\boldsymbol{g}_{i}^{\prime}}\right)
\end{gathered}
$$

where $\boldsymbol{X}_{t-1}^{\boldsymbol{g}_{i}^{\prime}}$ is the set of targets' states belonging to the groups in $\boldsymbol{g}_{i}^{\prime}$. The vector $\boldsymbol{z}_{t}^{\boldsymbol{g}_{i}}$ comprises the subset of measurements 
related with the group in $\boldsymbol{g}_{i}^{\prime}$. For example, $\boldsymbol{z}_{t}^{\boldsymbol{g}_{i}}$ can be chosen by gating measurements using the set of targets state $\boldsymbol{X}_{t-1}^{\boldsymbol{g}_{i}^{\prime}}$.

Algorithm 1 shows a particle filter algorithm developed from the Bayesian formulation above, where $N_{p}$ represents the number of particles.

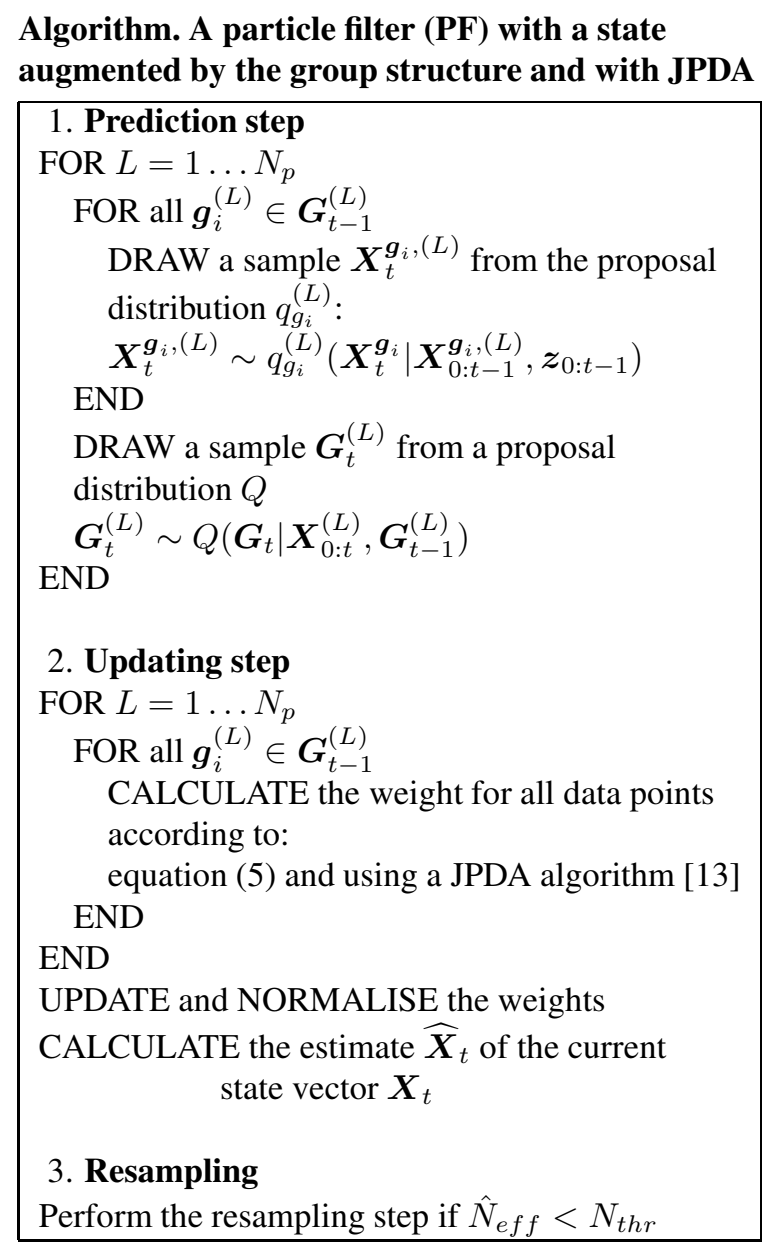

\section{TESTING RESULTS}

In this section, the proposed approach is validated over real GMTI data shown in Figure 2. These data are provided to us by QinetiQ [14]. Two groups of targets are moving on the ground and there is a crossing of their paths which makes additional ambiguity for the group tracking algorithm. The GMTI measurements are obtained by an embedded radar on a moving airborn platform. As seen from Figure 2, there is clutter noise in the measured bearing angles and measured distances to the targets. The developed approach provides good estimation accuracy of each vehicle trajectory positions (see Figure 3 ). Figure 4 additionally shows that the estimated $x$ coordinates of the groups are close to $x$ coordinates calculated from the measurements. The proposed algorithm is able to cope with the crossed trajectories of the groups. Addi-
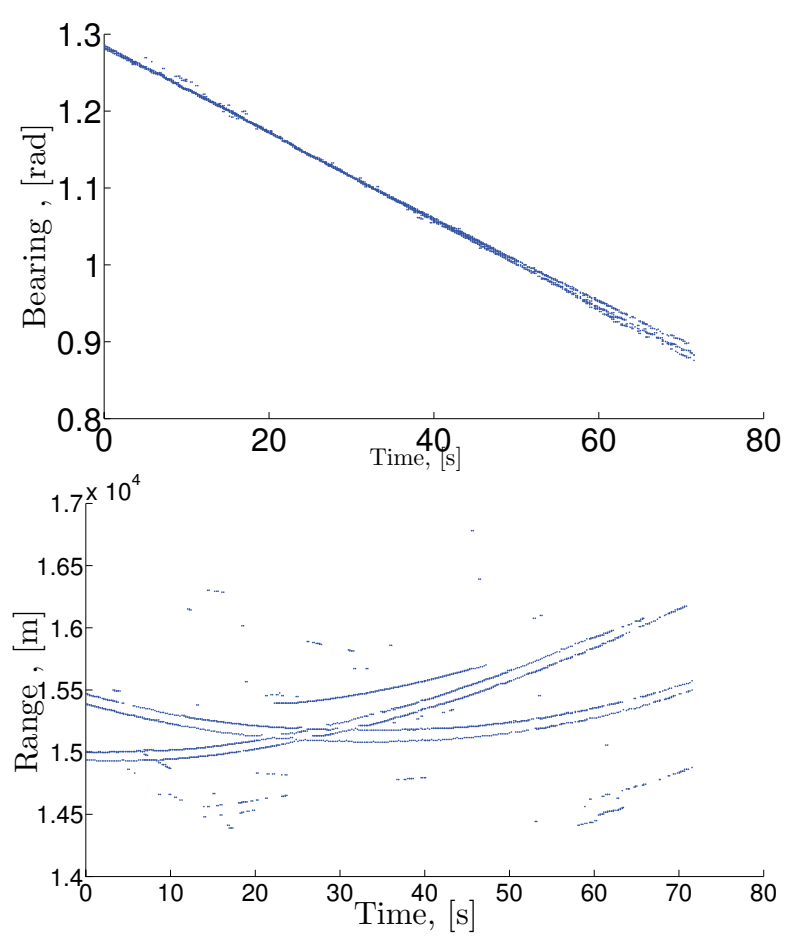

Fig. 2. Measured bearing and measured range, resp. for two groups.

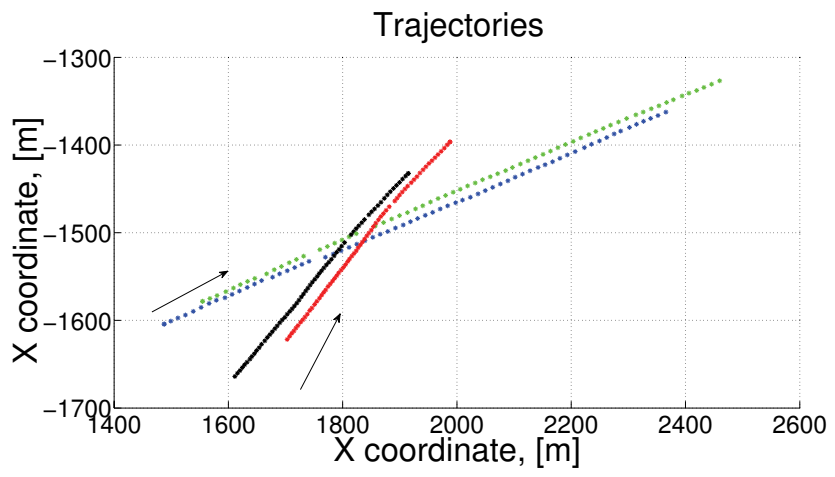

Fig. 3. Estimated trajectories for the 2 groups. The arrows show the directions of the movement

tionally, Figure 5 presents the group structures estimated by the particle filter. In the real scenario vehicles 1 and 2 are forming group 1 and vehicles 3 and 4 are forming the second group. To plot the Figure 5, only four relevant group structures appearing during the estimation process are labeled from 1 to 4 (respectively $\boldsymbol{G}_{1}:\left\{\boldsymbol{g}_{1}=(1,2), \boldsymbol{g}_{2}=(3,4)\right\}, \boldsymbol{G}_{2}$ : $\left\{\boldsymbol{g}_{1}=(1), \boldsymbol{g}_{2}=(2), \boldsymbol{g}_{3}=(3,4)\right\}, \boldsymbol{G}_{3}:\left\{\boldsymbol{g}_{1}=(1,2), \boldsymbol{g}_{2}=\right.$ $\left.(3), \boldsymbol{g}_{3}=(4)\right\}, \boldsymbol{G}_{4}:\left\{\boldsymbol{g}_{1}=(1), \boldsymbol{g}_{2}=(2), \boldsymbol{g}_{3}=(3), \boldsymbol{g}_{4}=\right.$ (4) $\}$ and a probability is calculated for each group at each time. From Figure 5 one can conclude that the group structure 


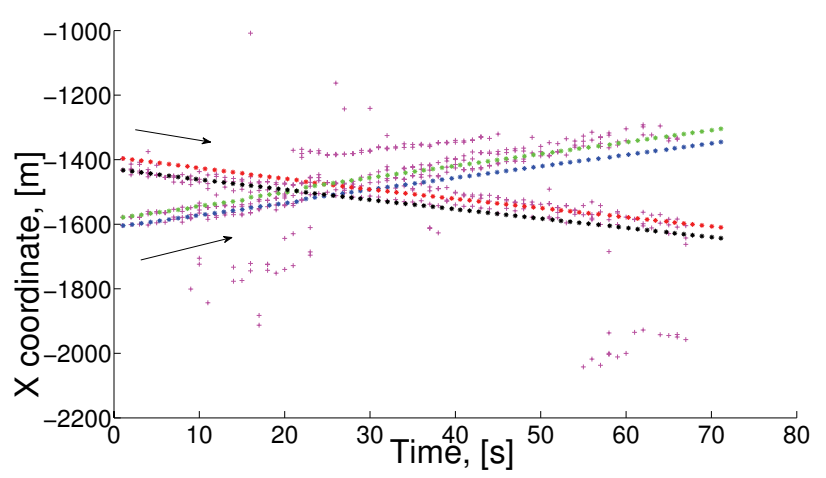

Fig. 4. Estimated $x$ coordinates for the 2 groups. The $x$ coordinates are calculated from the measurement and plotted too. The arrows show the directions of the movement.

is well estimated by the introduced graph evolution model. In addition, we can deduce precious information about the group structures uncertainty during the time evolution.

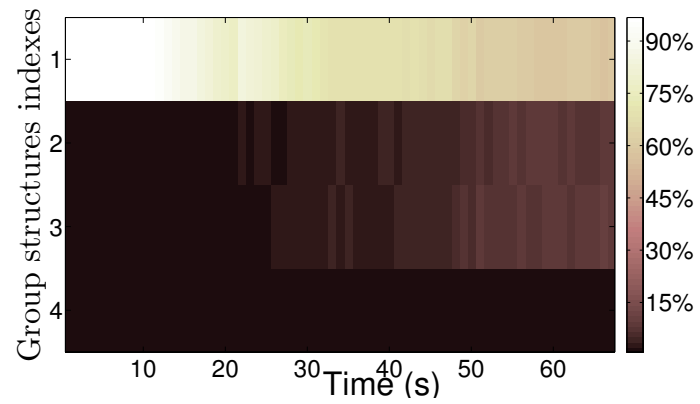

Fig. 5. Group structure evolution estimated by the PF. The 4 more relevant group structures are labeled from 1 to 4 and a probability is calculated, for each group and at each time step.

\section{CONCLUSIONS}

This paper presents a solution to the problem of group structure and state estimation in the presence of measurement origin uncertainty. The group structure is modeled as an evolving random graph and an evolution model is designed for the group structure. In addition, an algorithm is developed combining particle filtering and joint probability data association. The graph structure is estimated in a probabilistic way by introducing a group graph structure in the particle filter samples. performance of the approach is validated over real ground moving target indicator data sets.

\section{REFERENCES}

[1] A. Gning, L. Mihaylova, S. Maskell, S. K. Pang, and S. Godsill, "Ground target group structure and state es- timation with particle filtering," in Proc. of International Conf. on Information Fusion, Germany, Cologne, 2008.

[2] M. Ulmke and W. Koch, "Road-map assisted ground moving target tracking," IEEE Trans. AES, vol. 42, no. 4, pp. 1264-1274, 2006.

[3] A. Jadbabaie, J. Lin, and A.S. Morse, "Coordination of groups of mobile autonomous agents using nearest neighbor rules," IEEE Transactions on Automatic Control, vol. 48, no. 6, pp. 988 - 1001, 2003.

[4] J.A. Marshall, M.E. Brouke, and B.A. Francis, "A pursuit strategy for wheeled-vehicle formations," in Proc. of 42nd IEEE Conf. on Decision and Control, Vol. 3, 2003, pp. 2555-2560.

[5] H. Axelsson, A. Muhammad, and M. Egerstedt, "Autonomous formation switching for multiple, mobile robots," in Proc. of the IFAC Conf. on Analysis and Design of Hybrid Systems, 2003.

[6] D. Dimarogonas and K. Kyriakopoulos, "A connection between formation control and flocking behaviour in nonholonomic multiagent systems," in Proc. IEEE International Conf. on Robotics and Automation, 2006.

[7] R. Mahler, Statistical Multisource-multitarget Information Fusion, Artech House, Boston, 2007.

[8] T. Vicsek, A. Czirók, E. Ben-Jacob, I. Cohen, and O. Shochet, "Novel type of phase transition in a system of self-driven particles," Phys. Rev. Lett., vol. 75, no. 6, pp. 1226-1229, Aug 1995.

[9] D. Helbing, "Traffic and related self-driven manyparticle systems," Review of Modern Physics, vol. 73, pp. 1067-1141, 2002.

[10] M. J. Waxman and O. E. Drummond, "A bibliography of cluster (group) tracking," in Proceedings of the SPIE Signal and Data Processing of Small Targets, O. E. Drummond, Ed., Aug. 2004, vol. 5428, pp. 551-560.

[11] S. N. Dorogovtsev and J. F. F. Mendes, "Evolution of networks," Advances in Physics, vol. 51, pp. 10791187,2002

[12] R. Albert and A.-L. Barabsi, "Statistical mechanics of complex networks," Reviews of Modern Physics, vol. 74, no. 1, pp. 47-97, 2002.

[13] S. Blackman and R. Popoli, Design and Analysis of Modern Tracking Systems, Artech House Radar Library, 1999.

[14] S. Maskell, "DIF DTC tracking cluster: GMTI demonstration," March 2008. 